\title{
PENSAR EN LA EXCELENCIA EDUCATIVA EN LAS ESCUELAS NORMALES SUPERIORES: UNA MIRADA HISTÓRICA ${ }^{12}$
}

\author{
Thinking about educational excellence in the Superior \\ Normal Schools: A historical look \\ Yheny Lorena Pineda Rodríguez* \\ Yasaldez Eder Loaiza Zuluaga**
}

Pineda, Y.L. \& Loaiza, Y.E. (2016). Pensar en la excelencia educativa en las Escuelas Normales Superiores: Una mirada histórica. Revista PACA, 8, 51-74

\section{Resumen}

El siguiente trabajo histórico- educativo da cuenta de la forma como las Escuelas Normales Superiores desde el siglo XIX hasta principios del XXI, vivieron cuatro momentos coyunturales que justifican, a grandes rasgos, el devenir histórico educativo de estás instituciones en Colombia y,

\footnotetext{
${ }^{12}$ Este trabajo hace parte de la tesis doctoral de investigación "Prácticas pedagógicas que caracterizaron el quehacer del maestro en el Ciclo de Formación Complementaria de las Escuelas Normales de Manizales, Pereira y Armenia, 1994-2010" con el método histórico como trayecto que la fundamentó, a partir de fuentes y vestigios primarios hallados en el sector magisterial, con entrevistas y análisis documental como técnicas de recolección de información.

*Magister en Educación. Candidata a doctora en Educación, Becaria de Colciencias, doctorados nacionales convocatoria 757 de 2016 y 785 de 2017. Investigadora activa, grupo de investigación "Maestros y Contextos", Universidad de Caldas. Docente de investigación y pedagogía, Programa de Formación Complementaria- Escuela Normal Superior de Manizales. Email: yhelopi@gmail.com **Doctor en Ciencias de la Educación. Profesor Titular, Universidad de Caldas. Integrante del grupo de investigación Programa reconstrucción del tejido social en zonas del postconflicto en Colombia, de la Universidad de Caldas. Profesor Asociado Universidad Católica de Manizales.

Email: yasaldez@ucaldas.edu.co
} 
especialmente en el Eje Cafetero. En virtud de ello, se da cuenta de la relación con el sujeto del saber pedagógico, el maestro; las transformaciones pedagógico-legislativas que han dado origen, en estos laboratorios pedagógicos, a otras posibilidades de prácticas pedagógicas, las que, tal vez, trascienden y conciernen la actualidad de los maestros normalistas.

Este trabajo investigativo se adelantó teniendo como referente las etapas del método histórico: Heurísitca (identificación y selección de las fuentes); Doxografía (clasificación de la información); Etiología (interpretación de la información); y Síntesis Histórica (informe final), con el objetivo de dar cuenta del devenir histórico de las Escuelas Normales, las cuales se reconocen como instituciones encargadas de la formación de maestros para los primeros niveles. Dentro de los hallazgos se puede plantear que las Escuelas Normales Superiores como entidades para la formación de maestros de los primeros niveles de educación en Colombia, son instituciones que permiten comprender, identificar y determinar procesos de investigación científica de orden histórico, en virtud a que en dichas instituciones se pueden reconocer las principales problemáticas que afectan la formación de maestros en el país, a partir de la la interpretación histórica, teórica, práctica y experiencial de su quehacer para dar cuenta de su papel en el acto educativo, el cual es su campo de acción.

Palabras Clave: Escuelas Normales, Maestro, Saber Pedagógico, Historia, Legislación, Tendencias

\section{Abstract}

The following historical-educational work accounts for the way in which the Higher Normal Schools from the nineteenth century to the beginning of the twenty-first century experienced four conjunctural moments that justify, in broad strokes, the historical educational development of these institutions in Colombia and, especially in the Coffee Axis. By virtue of this, the relationship with the subject of pedagogical knowledge, the teacher, is realized; the pedagogical-legislative transformations that have given rise, in these pedagogical laboratories, to other possibilities of pedagogical practices, which, perhaps, transcend and concern the actuality of the normalist teachers. 
This research work was carried out with reference to the stages of the historical method: Heurisitca (identification and selection of sources); Doxography (classification of information); Etiology (interpretation of information); and Historical synthesis (final report), with the objective to give an account of the historical evolution of the Normal Schools, which are recognized as institutions responsible for the training of teachers for the first levels. Among the findings it can be stated that the Superior Normal Schools as entities for the training of teachers of the first levels of education in Colombia, are institutions that allow to understand, identify and determine scientific research processes of historical order; in virtue of the fact that these institutions can recognize the main problems that affect the training of teachers in the country, based on the historical, theoretical, practical and experiential interpretation of their work to account for their role in the educational act, which is its field of action.

Keywords: Normal Schools, Teacher, Pedagogical Knowledge, History, Legislation, Trends

\section{Introducción}

Las Escuelas Normales en Colombia han tenido como misión la formación de maestros para la educación básica primaria y, aunque han afrontado diversos hechos y acontecimientos producto de devenires políticos, religiosos, sociales, económicos y legislativos, han sabido sostener su ideal educativo, formar maestros para la educación preescolar y básica primaria, y han depositado un legado extendido hacia las nuevas generaciones sobre las características morales, académicas y pedagógicas que la sociedad les ha exigido.

Un primer momento es asumido desde la concepción de la educación como elemento cultural integrador y totalizador de significados que influye en las nacientes Normales en cada departamento de Colombia y, por ende, en la ciudad de Manizales, como capital de Caldas -Viejo Caldas, conformado por lo que actualmente se conoce como Caldas, Quindío y Risaralda-. Se da un segundo momento, hacia mediados del siglo XX, gracias a la incursión de la tecnología instruccional que favoreció la homogeneización de la educación con finalidades profundamente económicas viabilizadas desde la diversificación de la enseñanza con el 
ciclo vocacional de educación que reemplazó el título de maestro por el de bachiller pedagógico, al patrocinar un currículo a prueba de maestros.

Consecuencias de incursiones foráneas que trajeron para el maestro y las Normales un tercer momento, al verse obligadas a reaccionar frente al decretado Plan de experimentación curricular, y por ende, justificar, articular, crear y sostener una nueva corriente de pensamiento denominado Movimiento Pedagógico Nacional, apoyado, entre otros, por agremiaciones magisteriales, intelectuales y universitarias, con la intención de recuperar el rol del maestro y proponer acciones para fortalecer su formación.

Y finalmente, un cuarto momento, período cumbre en el que se cristalizan las acciones del Movimiento Pedagógico, se aprueba la Ley General 115 de 1994, se reestructuran las Normales en Escuelas Normales Superiores y se decreta el camino para pensar en la excelencia educativa con los procesos de acreditación previa y acreditación de calidad y desarrollo total en estas instituciones educativas.

Hasta el siglo XVIII todo futuro educador con condiciones personales de vocación y aptitud recibía educación junto a otro maestro en forma gremial; época a partir de la cual diferentes países europeos prepararon de manera especial los maestros, inicialmente en seminarios, como en Alemania, posteriormente en las Escuelas Normales -versión de aquellos- en Francia y, finalmente, en el siglo XIX, desplegadas las Normales por todo el mundo sobre la base de la enseñanza primaria con estudios que duraban de dos hasta cinco años, orientados como su nombre lo indica, en escuelas que daban la norma, la orientación pedagógica en la formación del magisterio (Luzurriaga: 1962, 275-276).

\section{Análisis y discusión}

En Colombia las Escuelas Normales nacen en el siglo XIX por la falta de institutores y la mejor opción para educarlos fueron las escuelas de oficios "puesto que el oficio del maestro era enseñar"; y como la enseñanza se consideraba un arte, había que estudiarla a través de la ciencia dispuesta para ello, la pedagogía, teoría trabajada de la mano de la práctica; con maestros considerados pedagogos, apóstoles, instructores de la juventud, con vocación, moral cristiana y política, pero además con fundamentos en aritmética, lectura, escritura e instrucciones en el método Lancasteriano 
o Pestalozziano (Álvarez: 1991, 139-158). Fue así como se materializó a través del Decreto general sobre instrucción pública del seis de octubre de 1820 como respuesta a las exigencias de una sociedad, frente a la necesidad apropiación de un saber primario, de la ciencia, con maestros que pudieran enseñar estos saberes y con centros adecuados para formarlos (Valencia: 2006, 40).

Luego de la independencia de Colombia y la separación de España hacia la segunda década del siglo XIX, se da un primer momento clave en el trasegar de las Escuelas Normales del país, puesto que, según Parra (1997, 21-22), para generar un elemento cultural integrador entre las identidades regionales dispersas, que aproximaran a la población hacia una idea identificadora de nación, de nacionalidad, dentro del contexto económico y político nacional, se concibió la educación como un elemento totalizador, como el mecanismo capaz de unificar significados, comprensiones y contenidos a ser transmitidos por los maestros de las Normales, de tal manera que todos los egresados, independientemente de la región del país a la que pertenecieran, adquirieran las mismas calidades y entrenamientos a ser propagados a futuro en sus comunidades; designios que, poco a poco, se desvirtuaron y supeditaron a intenciones más que culturales, a otras de tipo económico, especialmente después de la segunda mitad del siglo XX.

En el desarrollo histórico de las Escuelas Normales se destaca hacia principios del siglo XX la disposición del gobierno nacional colombiano con la Ley 39 de 1903, reglamentada por el Decreto 491 de 1904, que aprobó la existencia de una Escuela Normal para varones y otra para mujeres en cada ciudad capital de los departamentos de Colombia; según Valencia (2006: 97-100), instituciones encargadas de formar maestros más prácticos y pedagogos que eruditos, para las que se nombraron personas con diploma de maestros graduados en algunas de las Normales ya institucionalizadas del país, con buena conducta moral y social, católicos, cumplidores de sus deberes; o bien, personas que sin ser maestros, profesaran la religión católica y la buena conducta moral y social, iniciativa que floreció en el recién creado Departamento de Caldas ${ }^{13}$ con el

\footnotetext{
${ }^{13}$ Departamento de Caldas o Viejo Caldas en el siglo XX conformador por los actuales departamentos de Caldas, Quindío y Risaralda.
} 
nacimiento de la Escuela Normal de Varones de Manizales según el Decreto 131 de dos de febrero de 1909 y un año más tarde, con la Escuela Normal de Institutoras de Manizales según el Decreto 86 de 26 de enero de 1910 (Valencia: 2006, 97-114), Normales que han dependido a lo largo de más de cien años, en su trayecto histórico educativo de un "contexto de formulación externa" por parte de diferentes fuerzas económicas, sociales, pedagógicas y políticas (Gimeno: 2015, 37), pero que, en el momento más álgido de crisis educativa en el país, conquistan regional y nacionalmente colectividades con puntos de apoyo reflexivo desde el pensamiento sociocrítico frente a posturas hegemónicas del poder que afectan la educación. A principios del siglo XX, un maestro caldense era aquel que representaba la autoridad llena de saber, considerado instructor en el conocimiento científico y transmisor de conocimientos básicos de la enseñanza, de la cultura general, para entender y dar a conocer el movimiento científico de la actualidad, capaz de articular en su labor pedagógica las enseñanzas de sus predecesores naturales; quien comprendiera la importancia de su labor, con una conducta irreprochable reflejada en un comportamiento que superara su preparación intelectual, un modelo a seguir para todos los ciudadanos, con una relación armónica, puntual, con amor por el estudio y respetuoso en la asistencia a los distintos cultos y compromisos con la iglesia (Valencia, 2006, p. 72-89).

"El mejor maestro es el que dispone en el más alto grado de cualidades intelectuales y morales, el que de una parte posee más saber, método, claridad y vivacidad en la exposición, y de otra, es el más enérgico, el más devoto, de su misión, el más apegado a sus deberes, al mismo tiempo que el más afectuoso para sus alumnos" (De Alcántara, 2003, p. 268).

Es así como se considera un segundo momento en la historia de las Normales, con más y más fuerza desde la segunda mitad del siglo XX, gracias al cumplimiento de directrices de organismos de cooperación internacional, bajo preceptos de planificación y calidad de la educación con la tecnología instruccional a partir de la Tercera Misión Pedagógica Alemana, las que conjuntamente, mediante parámetros establecidos, orientan el Ministerio de Educación Nacional y, por ende, generan en la legislación educativa la tendencia hacia la masificación de la educación y posterior transformación de las Normales en el país, a partir de los Decretos 1710 y 1955 de 1963. 
La disposición económica de la época creó la necesidad de homogeneizar la población a partir de específicas formas de ver la realidad, desde significados, prácticas y contenidos de formación, desde la uniformidad de métodos de enseñanza, de ninguna manera con intenciones de unificación nacionalista, sino como elemento necesario para preparar recursos humanos que cubrieran la demanda de los empleadores en los sectores aplicados en la industria, la agricultura y el comercio (Parra: 1997, 167-169).

Por ello, el Ministerio de Educación Nacional, aprobó la figura institucional de la enseñanza media diversificada con el Ciclo Vocacional, de dos años, mediante el Decreto 080 (22-01-1974), con el que apoyó la renovación curricular para las Escuelas Normales del país, al definirse específicamente los discursos y acciones del maestro en la formación de los futuros maestros, con base en un plan de estudios de seis años con uno más de práctica para la nueva modalidad de bachillerato pedagógico, en consonancia con las modernas tendencias educativas y las necesidades del país; propuesta que se viabilizó con la Resolución 4785 de 1974, en la que se dio gran valor a la experimentación pedagógica, a las prácticas pedagógicas desde lo declarativo, desde los planes de estudios con miras a consolidar técnicas pedagógicas para el desarrollo eficiente de la práctica pedagógica (Loaiza: 2009, 75), pero que, sumado a otros factores contextuales futuros, propendieron por el menoscabo intelectual y cultural de la educación.

Hacia la década del setenta, se consideraba un maestro como aquella persona que enseñaba en una escuela primaria, guía erudito, cualitativamente distinto de lo abstracto y ausente de las técnicas audiovisuales; capaz de hacer revelar a los estudiantes sus aptitudes o ineptitudes, patrimonio hereditario, para posteriormente colocarlos en las mejores condiciones de ejercicio, permitirles desarrollarse al máximo y utilizarlos para lo mejor (Foulquie, 1976, p. 288-289).

Por otra parte, la Resolución 7126 de 1982 estableció el carácter experimental del Plan de Estudios normalista mediante el Plan de Experimentación Curricular y adoptó programas para el ciclo de básica secundaria y el ciclo de educación media vocacional; se reajustaron algunos programas, se elaboraron otros del área de pedagogía y se estructuró el Manual de Práctica Docente de acuerdo con la nueva 
concepción curricular de Educación Básica Primaria. Asimismo, las Escuelas Normales entraron a formar parte de los subproyectos nacionales cofinanciados con el Banco Mundial dentro del Plan de Fomento para áreas rurales y centros menores de población.

De esta manera, según Loaiza, Pineda y Arbeláez (2014, 259-280), la nueva legislación con medidas como educación programada, educación sin maestros, currículo a prueba de maestros, educación a distancia, autogestión comunitaria, autofinanciación, descentralización, entre otros, aunque conllevaron a la cobertura escolar, en absoluto resolvieron el problema de deterioro intelectual y cultural de la educación; más bien limitaron al maestro a ser simple administrador del currículo, funcionario, recurso o factor de desarrollo, quien debía aplicar métodos de enseñanza foráneos donde carecían del conocimiento contextual, la población, el ambiente escolar, las prácticas cotidianas, los espacios pedagógicos, su cultura, sus creencias, entre otros.

Panorama incierto y desalentador para los maestros del país quienes, entre los años ochenta y noventa del siglo XX en Colombia, decidieron afrontar la situación, asumir una postura socio-crítica de la educación, reconquistar el liderazgo cultural del maestro y democratizar las prácticas pedagógicas. Este período trascendental, tercer momento en la historia de las Normales, surge con la nueva corriente de pensamiento llamada Movimiento Pedagógico, con sus respectivas Expediciones Pedagógicas en diferentes departamentos del país; tránsito que posibilitó al menos tres tipos de prácticas pedagógicas de los formadores de maestros con base en otras posibilidades de contextualización pedagógico-académica, según los nuevos paradigmas ofrecidos por las facultades de educación y que por ende, conllevaron a su reflexión.

Movimiento en el que surgieron luchas y compromisos con la misión de formación de maestros para la educación básica primaria desde un contexto de formulación interna, desde saberes generados por los mismos maestros, inicialmente desde el magisterio de departamentos como Nariño, Antioquia, Huila y el Distrito Especial de Bogotá, quienes organizaron las primeras comisiones pedagógicas, elaboraron los primeros planes de trabajo y desarrollaron un proceso de estudio e investigación colectiva; y posteriormente, se sumaron comisiones creadas en los departamentos de 
Risaralda, Cauca, Caldas, entre otras (Loaiza, Pineda y Arbeláez: 2014, 75).

Entre las universidades, las facultades de educación y los movimientos intelectuales que más apoyaron el Movimiento Pedagógico en Colombia, estuvieron la Universidad de los Andes, la Universidad Nacional con el grupo Federici, compuesto por Antanas Mockus, Jorge Charum, José Granés, Clemencia Castro y Carlos Augusto Hernández; y la Universidad de Antioquia, con su centro de Investigaciones Educativas adjunto a la Facultad de Educación, compuesta por Olga Zuluaga, Alberto Echeverri, Rafael Flórez, Mario Gómez, José Iván Bedoya (Loaiza, Pineda y Arbeláez: 2014, 79).

Movimiento pedagógico nacional creado con el fin de cambiar el carácter administrativo-económico de la educación, defender las diferencias sociales por verdaderos ambientes pedagógicos; reconceptualizar los conocimientos producidos en el seno del saber pedagógico o de otros saberes acerca de la enseñanza y la educación; recuperar el rol cultural del maestro, su libertad como ciudadano pertenecientes a una sociedad democrática; dejar de ser ejecutores de las políticas estatales para transformarse en pensadores de su quehacer; generar procesos continuos de autocrítica sobre el trabajo diario, contextualizar el quehacer; crear estructuras renovadas del saber pedagógico y, según Flórez y Batista (1982: 158), construir modelos pedagógicos que, al partir de recursos y necesidades propias, formaran los ciudadanos del mañana con capacidad crítica y reflexiva.

Entes activos para luchar por la reivindicación profesional del maestro, quienes exteriorizaron de su conciencia colectiva, el valor del quehacer pedagógico, la renovación e intercambio de experiencias, la redefinición y transformación de la escuela, de procesos educativos y de la pedagogía como campo del saber y sus correspondientes consecuencias, con el fin de buscar soluciones para mejorarla; así como introdujeron para la discusión del maestro colombiano las teorías de Jürgen Habermas, Basil Bernstein, Thomas Samuel Kuhn y Michael Foucault, con nuevas corrientes de pensamiento sobre la naturaleza de la educación, la escuela, el papel y las tareas del educador (Loaiza, Pineda y Arbeláez: 2014, 81). 
Es importante reconocer que los aportes metodológicos de la filosofía de Michel Foucault fueron asumidos para aplicar arqueología a la producción de los discursos sobre la educación, desde la excavación de cómo emergieron éstos, puesto que el maestro tiene un saber, producto de la experiencia, que circula en todos los niveles educativos y que, por tanto, de ninguna manera es exclusivo de un solo individuo. "La arqueología, al dirigirse al espacio general del saber, a sus configuraciones y al modo de ser de las cosas que allí aparecen, [que proviene del conocimiento común, de la empiria, de lo cotidiano del maestro], define los sistemas de simultaneidad, lo mismo que la serie de las mutaciones necesarias y suficientes para circunscribir el umbral de una nueva positividad" (1968, 8); es así como pasaron de prácticas discursivas hacia formaciones discursivas, del saber, que cualquiera lo tiene, hacia el conocer desde el campo del conocimiento epistémico para acceder a la elaboración, desde las reglas del discurso, para abordar epistemológicamente el trabajo empírico.

Como lo afirma Larrosa (1995: 271), las prácticas pedagógicas son lugares de mediación en los que [el maestro] encuentra los recursos para el pleno desarrollo de su autoconciencia y de su autodeterminación, o para la restauración de una relación no distorsionada consigo mismo; espacios institucionalizados donde la verdadera naturaleza de la persona humana en tanto que autoconsciente y dueña de sí misma puede desarrollarse y/o recuperarse". Prácticas que simultáneamente configuraron modos de ser maestros, según la reflexividad de su experiencia, de los problemas de la realidad educativa, de las teorías educativas, pedagógicas y didácticas, de su propia práctica y de su lugar en el mundo laboral. Dinámicas que dispusieron prácticas pedagógicas repetitivas, prácticas pedagógicas entre lo tradicional y lo nuevo y prácticas pedagógicas totalmente separadas de la pedagogía y más cercanas a una disciplina académica específica (Zuluaga, 1987).

Movimiento que con el transcurrir de los hechos y acontecimientos políticos, económicos y educativos del país, se fortaleció en congresos, foros, eventos, conversatorios, seminarios, paneles de debate educativo, encuentros de instituciones formadoras de maestros, la Revista trimestral "Educación y Cultura", la radiodifusora "La voz de los educadores", donde se gestaron redes, colectivos pedagógicos y expediciones pedagógicas 
locales, regionales y nacionales para hacer diagnósticos de la crisis de la educación y la pedagogía, reflexionar y proponer alternativas a las políticas educativas con sus consecuentes reformas; deliberar sobre la reivindicación de los maestros y la reforma a las Escuelas Normales, con un sistema de formación especial (2014: 88; Mejía: 1987, 15-16; Rodríguez: 2002, 23). Expedición que para el caso específico del Eje Cafetero, se consolidó desde las Escuelas Normales, hasta congregar el conjunto del gremio magisterial en todos los niveles del sistema educativo, de la mano de sectores populares, quienes suscitaron públicamente construcciones colectivas y acuerdos sociales con el fin de devolverle la palabra a los sujetos del saber pedagógico, darles voz desde la experiencia, desde el quehacer cotidiano y desde el compartir de saberes para constituir un discurso pedagógico propio en las instituciones formadoras de maestros (Archivo Historial, Escuela Normal Superior de Manizales, 1984-1991). Reflexiones que ultimaron diagnósticos regionales particulares, en contextos reales, propios, con el fin de recuperar la identidad, la autonomía y el rol cultural del maestro y pasar de una realidad dada a otras nuevas realidades (Mejía, 1987, p. 39-40; Colectivo de autores, 1991. p. 28).

Análisis e interpretaciones, publicados localmente en la revista "Correo Pedagógico" del Centro de Estudios e Investigaciones Docentes -CEIDcon el apoyo de centros de documentación, del Sindicato de los Educadores Unidos de Caldas -EDUCAL- vinculados con la Federación Colombiana de Educadores -FECODE- y la revista "Educación y Cultura"; así como con la Asociación Caldense de Licenciados -ACAL- (Cárdenas, 2000, p. 37), el Sindicato de Trabajadores del Ministerio de Educación Nacional SINTRENAL-, la División General de Capacitación del Centro Experimental Piloto -CEP-, las Escuelas Normales del Departamento de Caldas y la Facultad de Educación de la Universidad de Caldas, quienes, en alguna medida, suscribieron espacios de discusión desde capacitaciones, comisiones y redes pedagógicas, grupos de estudio, microcentros, seminarios, foros, congresos y encuentros regionales de Escuelas Normales, de maestros e instituciones educativas (Archivo Historial, ENSM, 19851991).

"Hicimos demasiado, foros, eventos, recorrimos municipios discutiendo la propuesta y dimos muchos elementos para el equipo redactor de la ley, los maestros se movieron mucho... Caldas en eso 
siempre ha sido juiciosa. Cuando la ley general, muchos se quejaron porque hicimos una ley para trabajar más, no para hacer pereza, entonces decían: la unión sindical nos puso a trabajar más, dijimos: es que vamos a cambiar eso, el sistema que tenemos, es un sistema que no sirve a la realidad de hoy, porque la primera ley general de educación fue la Ley 39 de 1903, la del ministro de educación Antonio José Uribe y era para una Colombia rural, confesional, para un país atrasado, de artesanos y demás... la ley que construimos es par propender por el desarrollo científico, tecnológico, de avanzada, porque se enseñe lo mejor en cada momento" (W. García, comunicación personal, Educadores Unidos de Caldas).

En ese sentido, en su momento, los maestros participaron en debates argumentados de política y legislación educativa, autoritarismo estatal, reivindicaciones salariales y responsabilidades sindicales (Aguilar y Álvarez, 1989, p. 58-61), como hechos derivados de las décadas del sesenta y setenta que condujeron a la taylorización de la educación, limitación de la escuela; relegaron el maestro, sin voz ni decisión, a ser administrador del currículo, instrumentalizaron la enseñanza, atomizaron los planes de estudios, desvirtuaron el papel de la pedagogía en la formación, entre otros.

"Cualquiera podía ser maestro... eso era lleno de bachilleres, uno por lo menos ya había hecho una licenciatura y porque pasaba por palanca, por ser amigo del Secretario de Educación. Por vocación las que estudiaban en Normales, pero muchos por accidente y yo diría que la mayor parte por política y en mí una coincidencia, yo vi que los que salían primero conseguían trabajo en el magisterio, era una fuente de empleo" (M. Cuesta, comunicación personal, Escuela Normal Superior de Caldas).

Panorama que configura el primer tipo de prácticas pedagógicas al lograrlas repetitivas, mecánicas (Mejía, 1987, p. 27), transmisionistas, descontextualizadas, rutinarias, convergentes y reproductoras de formas sociales de dominación (Acevedo, 1985, p. 144-147); prácticas que, al ser poco reflexionadas, se naturalizaron, legitimaron y heredaron. 
"...Pues eran casi todos normalistas, luego se profesionalizaron aunque lo que estudiaron tal vez lo reflejaban en sus clases la primera semana pero luego seguían con el mismo cuaderno todos los años, hasta los estudiantes se burlaban de ellos y hacían chistes de eso, porque con el mismo cuaderno trabajaban toda la vida" (H. Parra, comunicación personal, ENSM).

Prácticas pedagógicas contempladas y reflexionadas por la expedición pedagógica de Caldas y sus afiliados, desde otras posibilidades, tanto desde las ciencias como desde las teorías, con aportes de la filosofía y de la sociología propuestos por el Movimiento Pedagógico, que suscitaron el segundo modo de ser maestro, una mixtura entre lo tradicional y los nuevos elementos (Archivo Historial, ENSM, 1984-1991).

Prácticas pedagógicas que emergieron como alternativas innovadoras desde el reconocimiento de diferentes procesos de formación entre lo urbano y lo rural, aunque con muchos vacíos epistémicos (Calonge y Quiceno, 1984) que, tal vez, mejoraron con la investigación pedagógica y construcción de saber pedagógico, ofertados desde las secretarías de educación (Archivo Historial, ENSM, 25-02-1989; Giraldo, 1991. p. 40-41) y desde la flexibilización del currículo para la formación de maestros en las instituciones formadoras de maestros (Acevedo, 1985, p. 82).

"Mario Díaz hace un estudio de las investigaciones en educación y muestra que las investigaciones en educación están marginadas de los maestros, o sea, están los investigadores por un lado y los maestros por otro, pero en realidad hay una conciencia de que la investigación tiene que ligarse a los maestros" (L, Herrera. comunicación personal, ENSM).

Y una tercera práctica pedagógica, viabilizada por el tránsito desde la vocación hacia la profesionalización en facultades de educación; para el caso particular de Caldas, la Resolución No. 029, aprobó el proyecto sobre cursos válidos como ascenso en el escalafón, en distintas modalidades en universidades de la región, a través de facultades de educación (Secretaría de Educación Municipal de Manizales, ENSM, 16-11-1982), en territorios particulares del saber, que fundamentaron la autoridad del docente de básica secundaria y media, con miras a recuperar su ser intelectual (Mejía, 
1987. p. 27), desde una ciencia o disciplina académica específica (Coral, 1985. p. 70), desde métodos de enseñanza con recursos didácticos provenientes éstas.

"En cambio el profesor como que llega, dicta su clase y se va, no alcanza a tener esa relación tan estrecha con los niños, ni a hacer un ejemplo tan patente como con la primaria" (O. Otálvaro, comunicación personal, ENSM).

Es así como maestros y profesores fueron, para este período histórico, considerados diferentes desde sus diversas formas de ser, saber y hacer que configuraron su práctica pedagógica; así la vocación, de la mano de la pedagogía y la formación axiológica del niño, caracterizaron los maestros de la básica primaria, mientras que los conocimientos de una ciencia o disciplina, caracterizaron los profesores. "El de primaria era el maestro, el de bachillerato es el profesor que llega dicta la actividad y se va, otra vez se queda sin conocer a los niños" (O. Otálvaro, comunicación personal, ENSM).

Se puede afirmar que en este tercer momento de las Escuelas Normales, las prácticas pedagógicas de los maestros formadores de maestros de ninguna manera fueron estáticas ni definitivas, más bien los nuevos retos y paradigmas que, con el transcurrir de los años ochenta y noventa ofrecieron las diferentes universidades y facultades de educación, conllevaron a su reconfiguración desde la reflexión de la experiencia pedagógica, desde lo que puede y debe ser pensado con miras al compromiso social, el pluralismo cultural y la contextualización objetiva del mundo educativo.

Y un último momento en la historia de las Normales, que inicia desde la promulgación del Decreto 1348 de 1990, que se concibe como fruto del Movimiento Pedagógico Nacional en el que se concibió la reforma las Escuelas Normales para adoptar un Sistema Especial de Formación de Maestros dentro de los principios constitucionales en orden a procurar el cumplimiento de los fines sociales de la cultura y la mejor formación intelectual, moral y física de los educadores. Decreto que, entre otros aspectos comprende ocho años de formación en una Escuela Normal, expedición del título de Maestro al finalizar y aprobar sus estudios con 
una opción de énfasis en su formación y dedicación exclusiva de las Escuelas Normales a la formación de Maestros.

Cuarto momento que se materializa finalmente con la Ley General de Educación 115 de 1994, el Decreto 3012 de 1997, la Ley 715 de 2001 y el Decreto 4790 de 2008 frente a la reestructuración y acreditación previa y de alta calidad del Ministerio de Educación Nacional, que compromete a las Escuelas Normales a enfrentar el contexto intelectual de las facultades de educación con miras a la capacitación y posterior fortalecimiento de procesos de investigación pedagógica-educativa, que otrora de ninguna manera existían o eran incipientes. Camino legislativo que estructuró un sistema integrado de desempeños del maestro en cuanto al discurso frente al saber pedagógico, didáctico y curricular y, por ende, desde lo declarativo; desde las realidades del saber evidenciadas en la acción, en lo procedimental, es decir, en la práctica pedagógica y desde los diversos escenarios pedagógicos institucionales, las que conjuntamente se regulan a partir de la normatividad educativa.

La Ley General de Educación (115 08-02-1994) con sus decretos reglamentarios señalaron, para todos los establecimientos educativos, elaborar y poner en práctica un Proyecto Educativo Institucional, PEI, que expresara la forma como se alcanzarían los fines de la educación definidos por la ley, según las condiciones sociales, económicas y culturales del medio. Igualmente declaró que las Escuelas Normales debían reestructurarse, aprobarse y autorizarse para formar educadores en el nivel de preescolar y en el ciclo de educación básica primaria; por lo tanto, debían operar como unidades de apoyo académico para la formación inicial de docentes y, mediante convenio celebrado con instituciones de educación superior, podían ofrecer formación complementaria que condujera al otorgamiento del título de normalista superior.

La formación de educadores a partir de esta ley fundó las bases para los fines y objetivos de la educación. Fue así como, el Decreto 0968 (09-061995) determinó la formación de educadores como un conjunto de procesos y estrategias orientados al mejoramiento continuo, de la calidad y el desempeño del docente, como profesional de la educación. Formación brindada en las Escuelas Normales Superiores, lo que la situó en el campo de preparación y capacitación permanente o en servicio, dirigido a 
educadores que se desempeñaran en el nivel de preescolar y en el ciclo de educación básica primaria.

Formación pedagógica que proporcionó para el gremio magisterial los fundamentos para el desarrollo de procesos cualificados integrales de enseñanza y aprendizaje, debidamente orientados y acordes con las expectativas sociales, culturales, colectivas y ambientales de la familia y de la sociedad; una formación disciplinar específica en un área del conocimiento, un saber o disciplina determinada o en la gestión de la educación; una formación científica e investigativa con los fundamentos y prácticas para la comprensión y aplicación científica del saber y la capacidad para innovar e investigar en el campo pedagógico; así como la formación deontológica y en valores humanos que promovieran la idoneidad ética del educador, de manera tal que contribuyera con los educandos, a la construcción permanente de niveles de convivencia, tolerancia, responsabilidad y democracia.

Dos años más tarde, el Decreto 3012 (19-12-1997), avaló como parte de su proceso curricular, la jornada única completa en las Escuelas Normales Superiores, en el nivel de educación media académica con profundización en el campo de la educación y la formación pedagógica y un ciclo complementario de formación docente con una duración de cuatro semestres académicos; con investigación pedagógica y planes de asistencia técnico-pedagógica para las instituciones educativas de su área de influencia, donde se incluyeron experiencias, contenidos y prácticas pedagógicas relacionadas con la atención educativa de las poblaciones de grupos étnicos, en el momento que elaboraron los correspondientes currículos y planes de estudio.

La acreditación de los programas de las Escuelas Normales en el proceso de reestructuración como Escuelas Normales Superiores se dividió en dos etapas; la inicial o acreditación previa, otorgada por un término de cuatro años, entre 1998 y 2002, tiempo en el cual el Ministerio de Educación Nacional reconoció la idoneidad y calidad de los programas académicos que ofrecían las Escuelas Normales Superiores y valoró la propuesta pedagógica de acuerdo con los niveles exigidos, como requisito para que las Secretarías de Educación les otorgaran la licencia de funcionamiento. El encargado de viabilizar el proceso de acreditación fue el Consejo de 
Acreditación de las Escuelas Normales Superiores -CAENS- que, entre otros, propuso como pilares para la certificación, primero, la fundamentación pedagógica sobre la necesidad de una tradición crítica en la construcción de un saber y sobre teorías, enfoques, modelos, escuelas de pensamiento y procesos de resignificación; segundo, la pedagogía, los núcleos del saber pedagógico y los núcleos del saber en relación con la formación profesional del educador; y, por último, la investigación educativa y pedagógica, las tendencias en la formación de maestros y la investigación en las escuelas normales superiores (Colectivo de autores: 2000).

Una vez que la Escuela Normal Superior se organizó y funcionó según lo propuesto, comenzó la segunda etapa de acreditación o acreditación de calidad y desarrollo, consistente en valorar y verificar, por parte del Ministerio de Educación Nacional, la puesta en marcha de la propuesta pedagógica de formación inicial de los maestros y, por ende, el cumplimiento de los requisitos de calidad de los programas ofrecidos y de desarrollo de los fines propios de la educación, acreditación otorgada por un período de cinco años (MEN, 2010; Decreto 3012: 1997).

La acreditación se inició primero con un plan de desarrollo a partir de la propuesta pedagógica de formación de maestros, con base en la cual se realizó la autoevaluación adelantada por cada Escuela Normal Superior, de acuerdo con los criterios determinados por el Consejo de Acreditación de las Escuelas Normales Superiores -CAENS-; es decir, se analizó cómo se habían materializado las diferentes dinámicas que la escuela normal propuso y los alcances concretos en la formación de los maestros. Segundo, con la evaluación externa practicada por un equipo evaluador nominado por el mismo Consejo y, por último, con la valoración que este realizó de los resultados de las dos primeras etapas y del respectivo proyecto educativo institucional.

Luego de la década de los noventa, las Escuelas Normales de Colombia se vieron en una situación de indeterminación, que dio lugar a diversas discusiones respecto a su naturaleza y pertinencia a tal punto de "no ser visibilizadas, es más pareciese que estuviesen ausentes"; situación que trató de subsanarse con base en las posibilidades que ofreció el campo de la pedagogía, los avances de la ciencia, la tecnología y las artes. Fue así como se recomiendó recoger la experiencia de la acreditación de calidad 
y desarrollo de las Escuelas Normales Superiores en los aspectos que garantizan la transformación efectiva de las prácticas de formación y que dieron lugar al desarrollo de experiencias significativas en la búsqueda de respuestas cada vez más acordes con las necesidades de la infancia y gestoras a su vez de otras formas de existencia (Baracaldo, 2007); experiencias que fueron más tarde centro de interés de múltiples investigaciones.

Correspondió a la Nación en materia de educación, según la Ley 715 (2112-2001), entre otro, definir normas, diseñar y establecer instrumentos y mecanismos para la calidad de la educación. así como establecer las normas técnicas curriculares y pedagógicas para los niveles de educación preescolar, básica y media, sin perjuicio de la autonomía de las instituciones educativas y de la especificidad de tipo regional. Aunque se afirmó que en América Latina los avances, propuestas y experiencias de reforma, mejora o modernización para la formación inicial de los docentes según las demandas sociales, tuvieron resultados negativos, según evaluaciones internacionales, lo que repercutió en el tema magisterial al responsabilizarlos directamente, lo que condujo a poner en tela de juicio su formación profesional (Flores, 2004).

Consecuentemente, el Ministerio de Educación Nacional aprobó el Decreto 1278 de 2002, que estableció el nuevo estatuto para el magisterio colombiano, que implicó en los procesos sistemáticos de enseñanza aprendizaje, el diagnóstico, la planificación, la ejecución y la evaluación de los mismos procesos y sus resultados, y de otras actividades educativas dentro del marco del proyecto educativo institucional de los establecimientos educativos. Función docente que, además de la asignación académica, comprendió actividades curriculares no lectivas, servicio de orientación estudiantil, atención a la comunidad; actividades de actualización y perfeccionamiento pedagógico; actividades de planeación y evaluación institucional, entre otras actividades formativas, culturales y deportivas, contempladas en el proyecto educativo institucional; y las actividades de dirección, planeación, coordinación, evaluación, administración y programación relacionadas directamente con el proceso educativo. Fue así como se denominaron genéricamente educadores a docentes y directivos docentes. 
Entre tanto, el artículo cinco del mismo decreto, reconoció como docente la persona que desarrolla labores académicas, directa y personalmente con los estudiantes de establecimientos educativos en un proceso enseñanza aprendizaje; responsables de las actividades curriculares no lectivas complementarias de la función docente de aula, entendidas como administración del proceso educativo, preparación de su tarea académica, investigación de asuntos pedagógicos, evaluación, calificación, planeación, disciplina y formación de los alumnos, reuniones de profesores, dirección de grupo, actividades formativas, culturales y deportivas, atención a los padres de familia y acudientes, servicio de orientación estudiantil y actividades vinculadas con organismos o instituciones del sector que incidieran directa o indirectamente en la educación.

El Artículo 40 del mismo decreto reglamentó, además, el marco ético de la profesión docente desde la comprensión de la educación como bien público, como actividad centrada en los estudiantes y al servicio de la Nación y de la sociedad; así, implicó una práctica que exigió idoneidad académica y moral, posibilitó el desarrollo y crecimiento personal y social del educador y del educando y requirió compromiso con los diversos contextos socio culturales en los cuales se realizaría.

Es así como el artículo 41 reglamentó como deberes de los docentes, la búsqueda permanente del incremento de la calidad del proceso de enseñanza - aprendizaje y sus resultados, mediante la investigación, la innovación y el mejoramiento continuo, de acuerdo con el plan de desarrollo educativo de la correspondiente entidad territorial y el Proyecto Educativo Institucional del establecimiento donde laboraría; además, educar a los alumnos en los principios democráticos, en el respeto a la ley y a las instituciones, en el amor a los valores históricos y culturales de la Nación; en conclusión, le correspondía observar una conducta acorde con la función educativa y con los fines, objetivos, derechos, principios y criterios establecidos en la Ley General de Educación y en los planes educativos (Decreto 1278, 2002).

Fue así como, visto desde la formación como elemento clave, se aseguró que quienes egresaban de las Escuelas Normales Superiores, sujetas a los referentes de calidad dispuestos por el Ministerio de Educación Nacional (MEN), debían caracterizarse principalmente por cuatro pilares que aseguraban su calidad, pilares de calidad que debían cumplir con 
condiciones de exhaustividad, relevancia, comparación entre instituciones educativas con fines de enriquecimiento mutuo.

Primero, la pedagogía como disciplina fundante, en la que el maestro, a partir de la comprensión de la realidad, es capaz de reflexionar, evaluar y proyectar su práctica pedagógica, en constante desarrollo, desde la complejidad del fenómeno educativo, para resignificarla, desde la investigación pedagógico educativa, dentro de las lógicas epistémicas como institucionales contextualmente propuestas (Colectivo de autores, 2000, p. 28).

Segundo, los núcleos del saber pedagógico que dan identidad profesional a los maestros y responden a la simbiosis entre educabilidad y enseñabilidad en una relación de práctica pedagógico-didáctica. Tercero, la investigación educativa desde la ontología, la epistemología y la metodología del conocimiento en el ámbito educativo, tanto por los maestros en formación en cuanto ser y quehacer de la profesión, así como de "maestros y directivos mediante líneas y proyectos de investigación que les permitan comprender su organización y el rol de sus actores, problematizar su propuesta de formación, desarrollar alternativas pedagógicas y realizar los procesos evaluativos necesarios para garantizar la calidad de sus servicios". Y, finalmente, la gestión educativa institucional en su dimensión formativa y en su relación con el contexto, con miras a la construcción del proyecto de Nación que las compromete (Colectivo de autores, 2000, p. 30-33).

Finalmente se puede decir entonces que la realización progresiva de las condiciones óptimas de una propuesta de formación en las Escuelas Normales de Colombia, evidenciadas en la evaluación, desde la autoevaluación y la evaluación externa, como aproximación al conocimiento de la calidad de los procesos y su evolución, fueron certificadas en el proceso de acreditación, con miras a validar, desde el Proyecto Educativo Institucional (PEI), la acción social portadora de progreso y desarrollo para el país; condiciones que implicaron para estas instituciones formadoras de maestros, una conciencia de autonomía colectiva que garantizara la autogestión de procesos de investigación y de construcción de saber pedagógico, como condiciones indispensables para sostener públicamente su nivel de calidad (Colectivo de autores, 2000, p. 7-9). 


\section{Conclusiones}

En el marco del sistema de aseguramiento de la calidad de la educación y con el objeto establecer las condiciones básicas de calidad para la organización y el funcionamiento del Programa de Formación Complementaria de Educadores, para el nivel de preescolar y el ciclo de básica primaria que ofrece una Escuela Normal Superior, se aprobó el Decreto 4790 (19-12-2008), decreto que posibilitó entonces articular el programa de formación complementaria de la Escuela Normal y el Proyecto Educativo Institucional -PEI- con principios pedagógicos de educabilidad, enseñabilidad, pedagogía y contextos, en el diseño y desarrollo de su propuesta curricular y plan de estudios y que dio los fundamentos para la dinámica académico-curricular de las Escuelas Normales.

Las Escuelas Normales en Colombia de ninguna manera han sido ajenas a los momentos coyunturales económicos, legislativos y educativos que han justificado los fines y contenidos de la educación desde las políticas educativas; han sabido afrontar, en las últimas décadas del siglo XX Y primeros del siglo XXI, con actitud reflexiva por parte de los maestros, la realidad de las pretensiones económicas, desde el pensamiento sociocrítico y han movilizado la población a tal punto de ser capaces de intervenir en las decisiones del gobierno a favor de la educación. Se puede afirmar, por tanto, que el diálogo, la socialización de la reflexión en la acción y de la acción, han generado saber pedagógico que cualifica, cada vez más, el trabajo del maestro y, por ende, forja el camino para pensar en la excelencia educativa de las Escuelas Normales Superiores.

\section{Referentes Bibliográficos}

- Acevedo C., Jairo (1985). Praxis y educación: un ensayo sobre praxis pedagógica, formación docente y educación popular. Medellín.

- Aguilar, Juan Francisco y Álvarez, Alejandro (1989). Los nuevos caminos del Movimiento Pedagógico. Revista Educación y Cultura. No. 19, FECODE, diciembre.

- Álvarez Gallego, Alejandro (diciembre, 1991). El Maestro: Historia de un Oficio. En: Revista Colombiana de Educación. CIUP-Universidad Pedagógica Nacional. No. 22-23, p. 139-158.

- Archivo Historial Escuela Normal Superior de Caldas. 
- Archivo Historial Escuela Normal Superior de Manizales.

- Baracaldo Quintero, Martha Elena (2007). "Escuelas normales superiores en el marco de la Visión 2019". Revista Javeriana 74, No.733: 38-45.

- Calonge Calonge, Patricia y Quiceno, Humberto (1984). El maestro marginal, una historia por escribirse. Revista Educación y Cultura. No. 2, FECODE, septiembre.

- Cárdenas Agudelo, Stella (2000). Construyendo saber pedagógico. Nodos y Nudos, Enredes, enero-julio.

- Colectivo de Autores (1991). La educación colombiana hacia el siglo XXI: perspectivas del Movimiento Pedagógico. Memorias del XI Seminario Nacional de Educación y Sociedad. Reflexión Educativa, Bogotá: CEPECS, Centro de Promoción Ecuménica y Comunicación Social.

- Colectivo de Autores, (2000), Formación de maestros, elementos para el debate. Consejo Nacional de Acreditación de Escuelas Normales Superiores, CAENS. Santafé de Bogotá.

- Coral, Laureano (1985). Lo social en la práctica pedagógica. Bogotá: Ediciones Ceis.

- De Alcántara García, Pedro (2003). Diccionario, Compendio de pedagogía teórico-práctica. [citando a Compairé, 1914]. Biblioteca virtual universal.

- Decreto 080 (22-01-1974), por el cual se deroga el Decreto 045 de 1962 y se dictan otras disposiciones sobre Educación Media. Presidente de la República, Misael Pastrana Borrero, Ministro de Educación Nacional, Juan Jacobo Muñoz Delgado. Diario Oficial 34038 (11-03-1974).

- Decreto 3012 (19-12-1997), por el cual se adoptan disposiciones para la organización y el funcionamiento de las Escuelas Normales Superiores.

- Decreto número 0968 (09-06-1995), por el cual se modifica el Decreto 2903 de 1994 que establece los procedimientos y condiciones para la reestructuración de las escuelas normales. Ministerio de Educación Nacional, presidente de la República. Diario Oficial Número 41884 viernes 9 de junio de 1995.

- Decreto número 4790 (19-12-2008), Por el cual se establecen las condiciones básicas de calidad del programa de formación complementaria de las escuelas normales superiores y se dictan otras disposiciones.

- Educación de Calidad, el camino para la prosperidad (2010). Escuelas 
Normales Superiores Acreditadas. Ministerio de Educación Nacional, República de Colombia. Visto en: http://www.mineducacion.gov.co/ 1621/articles-208800_archivo_pdf_ens_acreditadas_junio2_2011.pdf

- Flores Arévalo, Isabel (2004). ¿Cómo estamos formando a los maestros en América Latina? Programa de Educación Básica de la Cooperación Alemana al Desarrollo (PROEDUCA), GTZ. Lima: Oficina Regional de Educación de la UNESCO para América Latina y el Caribe.

- Flórez Ochoa, Rafael y Batista Jiménez, Enrique (1982). El pensamiento pedagógico de los maestros de educación primaria oficial de Medellín. Copiyepes: Medellín.

- Foucault, M. (1968). Las palabras y las cosas: una arqueología de las ciencias humanas. Siglo XXI.

- Foulquie, Paul (1976). Diccionario de pedagogía. Barcelona-España: oikos- tau.

- Gimeno Sacristán, José; Santos Guerra, Miguel Ángel; Torres Santomé, Jurjo; Jackson, Philip W.; Marrero Acosta, Javier (2015). Ensayos sobre el currículum: Teoría y práctica. Primera parte, Perspectivas Contemporáneas sobre el Currículum. Capítulo II: El currículum como estudio del contenido de la enseñanza. España, Universidad de Valencia: Ediciones Morata, S. L.

- Giraldo Arias, Ofelia (1991). Influencia socio-cultural de la Normal de la Presentación en el municipio de Pensilvania, Caldas. Tesis, Universidad de la Sabana. Facultad de Educación. Programa de Ciencias Sociales, Bogotá.

- H. Parra, comunicación personal, Escuela Normal Superior de Manizales.

- L, Herrera. comunicación personal, Escuela Normal Superior de Manizales.

- Larrosa, J., Díaz, M., Donald, J., Hunter, I., Varela, J., \& Walkerdine, V. (1995). Escuela, poder y subjetivación. La Piqueta.

- Ley 0715 (21-12-2001), Por la cual se dictan normas orgánicas en materia de recursos y competencias de conformidad con los artículos 151, 288, 356 y 357 (Acto Legislativo 01 de 2001) de la Constitución Política y se dictan otras disposiciones para organizar la prestación de los servicios de educación y salud, entre otros.

- Ley 115 (08-02-1994). Por la cual se expide la Ley General de Educación.

- Loaiza Zuluaga, Yasaldez; Pineda Rodríguez, Yheny Lorena y Arbeláez J., Natalia. (2014). Libro: Debate pedagógico, el maestro de las Escuelas Normales de Manizales en el contexto del Movimiento Pedagógico 
Colombiano. Manizales: Universidad de Caldas.

- Loaiza Zuluaga, Yasaldez Éder. (2009). Sabio o Erudito: El maestro de las escuelas normales del Departamento de Caldas 1963- 1978. Manizales: Universidad de Caldas. Libro de Investigación No. 33.

- Luzurriaga, Lorenzo. (1962). Diccionario de pedagogía. Buenos Aires: Losada.

- M. Cuesta, comunicación personal, Escuela Normal Superior de Caldas.

- Mejía J., Marco Raúl (1987). Movimiento Pedagógico. Una búsqueda plural de los educadores colombianos. Documentos ocasión. Bogotá: CINEP.

- Ministerio de Educación Nacional (2010). Escuelas Normales Superiores Acreditadas. Educación de Calidad, el camino para la prosperidad. Ministerio de Educación Nacional, República de Colombia. Visto en: h t t p : / / w w w. mineducacion.gov.co/1621/articles 208800_archivo_pdf_ens_acreditadas_junio2_2011.pdf

- Otálvaro, comunicación personal, Escuela Normal Superior de Manizales.

- Parra Sandoval, Rodrigo (1997). Los maestros Colombianos. Bogotá: Plaza y Janes.

- Rodríguez Céspedes, Abel (2002). Movimiento Pedagógico: un encuentro de los maestros con la pedagogía. En: Veinte años del Movimiento Pedagógico: 1982-2002, entre mitos y realidades. Bogotá: Cooperativa Editorial Magisterio.

- Valencia Calvo, Carlos Hernando, (2006). Las Escuelas Normales y la Formación del Magisterio, Manizales Primera mitad del siglo XX. Colombia: Universidad de Caldas.

- W. García, comunicación personal, Educadores Unidos de Caldas.

- Zuluaga de Echeverry, Olga Lucía (1987). Pedagogía e historia, la historicidad de la pedagogía. La enseñanza, un objeto de saber. Foro Nacional por Colombia: Bogotá. 